\title{
Telescopic displays
}

\author{
Liquid-crystal displays are hugely successful in today's world, but their back-light transmission \\ efficiency is relatively small. Nature Photonics spoke to Anna Pyayt, who, with colleagues at \\ Microsoft, has devised a display approach that could offer improved light efficiency at lower cost.
}

Tell us about your new display technology. Our aim was to create a display that can offer better light efficiency than existing technologies. To do this we used a new optical design, in which each pixel acts like a miniature telescope. This leads to a more light-efficient display. The idea is fairly similar to that behind a Newtonian telescope.

How does the telescopic pixel design work? Instead of using a traditional on-off shutter system to transmit light, we use two metal mirrors - a primary mirror, which is deformable, and a secondary mirror, which is stationary. When the pixel is in an off-state both mirrors are flat, and they basically just stop light and reflect it back.

When we apply a voltage between a transparent electrode and the primary mirror, the mirror deflects towards the electrode, giving it a shape that is close to parabolic. So instead of reflecting the light back, the primary mirror focuses light onto the secondary mirror. This light is then reflected from the secondary mirror back through a hole in the primary mirror onto a screen. It's a pretty simple concept.

\section{How big is your device?}

The primary mirror is $100 \mu \mathrm{m}$ in diameter, which basically determines the size of the pixel. The separation between the primary and secondary mirrors is $180 \mu \mathrm{m}$. The overall prototype is several centimetres in diameter.

What is the device performance like? Our first prototype system offers a backlight transmission efficiency of $36 \%$, which is several times better than the comparable efficiency of LCDs (about 5-10\%). The improvem ent stems from the fact that we use just two mirrors, whereas LCDs require many functional layers, each of which can stop a large amount of light from passing through. Theoretically, our telescopic design can reach efficiencies of $50 \%$, although we would have to improve our fabrication technique to get closer to that number.

\section{How easy is it to produce your} pixel displays?

Fabrication is pretty straightforward as it involves a small number of steps. We use just

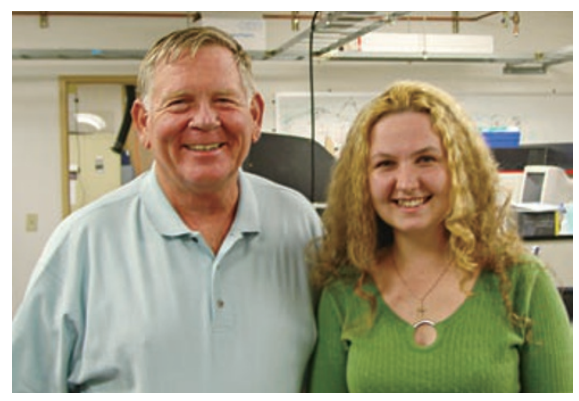

Gary Starkweather, co-author and also inventor of the laser printer, and Anna Pyayt.

a few layers - two metal layers, one layer of transparent conductor (ITO) and a spacer layer that can be made from plastic. Each layer is made from very stable, standard materials, which is actually an important advantage because material degradation can be a problem for many types of displays. The fabrication technique we use is compatible with an LCD production line.

What about the contrast of the display? The contrast of our prototype is only 20:1 at present, but this figure was obtained using non-collimated backlight. Our simulations show that we could achieve contrast ratios of up to 800:1 using collimated light, but this will require additional work.

Your co-authors are based at Microsoft. How did you get involved with them? I was familiar with some of the displays work that Microsoft was doing. I really wanted to work with them so I basically contacted them and applied for an internship. They gave me the position, which lasted three months, and during that time I worked with Gary Starkweather (inventor of the laser printer) and Michael Sinclair. At the end of it we continued working together, and Microsoft is actually fully funding my $\mathrm{PhD}$ at the University of Washington.

Aside from light efficiency, what are the advantages of your display approach? For a start, the system is very easy to operate. The pixels can be scaled up or down in size, which makes them potentially useful for high-resolution displays. The technology also lends itself to modularization, in which many small display modules are combined together to form large displays. When the size of a display is increased, its fabrication cost increases dramatically because the number of fabrication defects increases in proportion to the surface area. Modular displays are less susceptible to defect issues and are cheaper to produce. So far, attempts to apply modular approaches to transmissive display technologies have resulted in displays with lower light efficiency and contrast than regular LCDs. Our technology could make progress on this front.

What kind of applications do you envisage your displays being used for?

I think our displays could be used to create brighter computer displays. But they also have the potential to drive forward low-cost, large screens for televisions and other applications. The consumer market is crying out for affordable, large, high-resolution televisions and displays. Telescopic pixel technology could lead to cheaper reflective or brighter transmissive modules for very large displays.

How much are your displays going to cost? At this stage - essentially the first demonstration of the technology - it's hard to predict the cost of the final commercial product. The current fabrication process is not only LCD-compatible but it also involves a smaller number of steps and lower-cost materials. Ideally, we would like our display to be significantly cheaper than the LCD.

\section{Where next?}

We would like to optimize different parameters of the new technology to achieve practical functionality. There is a big difference between offering the first demonstration of the technology and producing a commercial device. I believe that many people will benefit if it finally happens.

Interview by Amber Jenkins.

Anna Pyayt, Gary Starkweather and Michael Sinclair have a Letter on their telescopic pixel display on page 492 of this issue. 